\title{
Dictadura Chilena y Sistema Escolar: "a otros dieron de verdad esa cosa llamada ${ }^{1}$ educación" ${ }^{2}$
}

\section{Ditadura chilena e sistema escolar: "aos outros foi dado realmente essa coisa que é chamada de educação"}

\section{Chilean dictatorship and School system: "education: that thing that was given to others"}

\author{
Alberto Moreno-Doña ${ }^{3}$ \\ Rodrigo Gamboa Jiménez ${ }^{3}$
}

\section{RESUMEN}

El trabajo que a continuación se presenta es una descripción de las políticas educativas que emergieron en Chile desde el inicio de la dictadura militar, cómo se consolidaron durante la vuelta a la democracia y las consecuencias que ellas han tenido para el sistema educativo-escolar actual. La instalación, con el inicio de la Dictadura Militar (1973-1990), de una lógica social, económica, cultural y educativa mercantilista impuesta por el General Augusto Pinochet ha determinado el presente actual, dificultando e imposibilitando

1 Este artículo se ha realizado en el contexto y gracias al Proyecto de Investigación Fondecyt N. ${ }^{\circ} 11110016$, titulado "La Educación Física y su función de transformación de las desigualdades sociales: profesorado del área y documentación ministerial”, financiado por el Fondo Nacional de Investigación Científica y Tecnológica de Chile.

2 El subtítulo del artículo es parte de la letra de una canción de la banda de rock chilena "Los Prisioneros" (para más información visitar http://es.wikipedia.org/wiki/Los_Prisioneros).

3 Pontificia Universidad Católica de Valparaíso. Viña del Mar, Valparaíso, Chile. Avda. El Bosque, n. ${ }^{\circ} 1290$ - Santa Inés. 
cambios profundos en la estructura de la institución escolar que permitan paliar las desigualdades educativas presentes en el país. Chile posee un sistema educativo segregacionista traducido en escuelas para los pobres, la clase media y los ricos, situación que ha sido denunciada por organismos internacionales. A pesar de los esfuerzos realizados por la Concertación de Partidos por la Democracia por mejorar la desigualdad existente, todas las propuestas han ido dirigidas hacia aspectos que no han permitido transformar la estructura que posibilita y legitima la desigualdad comentada.

Palabras clave: dictadura chilena; políticas educativas; desigualdad.

\title{
RESUMO
}

O trabalho apresentado a seguir é uma descrição das políticas educacionais que surgiram no Chile desde o início da ditadura militar, como foram consolidadas durante o retorno à democracia e as consequências que têm sobre o atual sistema de ensino da escola. A instalação, com o início da ditadura militar (1973-1990), de uma lógica social, econômica, cultural e educacional mercantilista imposta pelo general Augusto Pinochet determinou o presente real, prevenindo e impedindo mudanças profundas na estrutura do sistema escolar para ajudar a combater as desigualdades educacionais no país. $\mathrm{O}$ Chile tem um sistema educacional apartheid que resultou em escolas para os pobres, para a classe média e para os ricos, uma situação que tem sido denunciada por organizações internacionais. Apesar dos esforços da Coalizão de Partidos pela Democracia para melhorar a desigualdade existente, todas as propostas têm sido dirigidas para as questões que têm impedido transformar a estrutura que permite e legitima a desigualdade discutida.

Palavras-chave: ditadura chilena; políticas de educação; desigualdade.

\begin{abstract}
The following article is a description of the educational policies that started to be followed in Chile from the beginning of the military dictatorship. It also explains how these policies became consolidated during the return to democracy and the consequences for the current school educational system. The starting imposition, by Augusto Pinochet (Military dictatorship, 19731990), of a mercantilist logic for society, education, culture and economy has shaped the current situation we are in. This imposition makes it very difficult for changes to happen in the school system (changes that would allow reducing the educational inequalities present in the country). International organisms have denounced that Chile has a discriminatory educational system, with schools for the poor, schools for the middle class and schools for the rich. Despite the efforts carried out by the "Concertación de
\end{abstract}


Partidos por la Democracia" (Coalition of Parties for Democracy) in order to improve this situation, all proposals have been focused on aspects that have not allowed a transformation of the structure that currently supports the above mentioned inequalities.

Keywords: Chilean dictatorship; educational policies; inequality.

\section{Dictadura militar en Chile y nuevas políticas educativas}

La transformación de las políticas educativas a raíz del inicio de la dictadura militar chilena en el año 1973 coincide con la implantación de un grotesco sistema económico neoliberal que permeó, y sigue haciéndolo, dichas políticas y el sistema educativo-escolar que de ellas se desprende (NÚÑ̃Z et al., 1984; RUIZ, 2010; RIESCO, 2007; CORVALÁN, 2013).

A partir de 1973, “[...] se realiza la mayor reforma en la historia de la política educativa chilena” (OLIVA, 2010, p. 312). Los cambios realizados por este régimen fueron radicales, totalizantes y generalizantes, comparables a los del presidente Frei Montalba y Salvador Allende, pero enmarcados e impregnados de ideologías neoliberales y de nacionalismo autoritario (NÚÑEZ, 1995).

Los cambios impuestos constituyen el desmantelamiento de las políticas y del proyecto educativo democratizador propuesto por el gobierno de la Unidad Popular (Escuela Nacional Unificada) que se proponía terminar con la desigualdad social reproducida en el sistema escolar y que reflejaba el fuerte compromiso del Estado por una educación pública y de calidad. Si bien ésta no logró ser implementada, es de relevancia destacar el proceso de concientización que generó en un gran sector del magisterio (RUIZ, 2010). No hay duda de que este ideal educativo viene de larga data en Chile, enfatizándose en las décadas comprendidas entre los años 1940-1970, pero se vio alterada por dramáticos cambios realizados por el Régimen Militar (CORVALÁN, 2013).

Estas aterradoras transformaciones pueden ser descritas en dos grandes periodos. El primero (1973-1979) se caracterizó por una fuerte desarticulación de la estructura educativa, acompañada de represión, control y eliminación de los sindicatos docentes, el gremio profesional, las agrupaciones estudiantiles y todas aquellas organizaciones profesionales y educativas. El segundo (1980-1990) se focalizó en la descentralización y privatización del sistema educativo, proceso basado en la lógica del sistema socio-económico de mercado imperante (RUIZ, 1997; CORVALÁN, 2013). Una clara señal que refleja lo ocurrido en estos dos periodos está relacionada con la subordinación del Ministerio de Educación al 
Ministerio del Interior (1973-1979) y al Ministerio de Hacienda en la segunda fase (CASTRO-PAREDES, 2012), situación ésta que describimos, con más detalles, a continuación.

En 1973 el Ministerio de Educación y las instituciones escolares quedan bajo el mando de la Armada y el Ministerio del Interior, quienes comienzan una profunda revisión del Currículo Escolar Nacional incorporando, en él, una lógica focalizada en principios nacionalistas, dando así inicio a un fuerte proceso ideologizante de todo sistema educativo. Estos cambios estructurales fueron acompañados de una notable disminución del presupuesto fiscal para el área en cuestión (RUIZ, 1997, 2010; CORVALÁN, 2013).

El Gobierno Militar, para asegurar el éxito de los cambios propuestos, inicia una campaña de represión sobre el colectivo docente, declarando ilegales a las institucionales gremiales. En 1974 se crea el Colegio de Profesores de Chile (Decreto 678/1974), con el propósito de obtener mayor control sobre dicho colectivo, quedando éste bajo la tutela de un grupo radical derechista designado por las autoridades militares. Conjuntamente con lo anterior, de las Escuelas Normales, encargadas de la formación de profesores hasta ese momento, fueron exonerados una gran cantidad de profesores/as, quedando entonces bajo la responsabilidad de personas leales al Régimen Militar (CAZANGA, 2013; RUIZ, 1997).

Se implementó, entonces, un aparato de espionaje, persecución y represión de las organizaciones políticas-educativas a través de los organismos del Estado. La Central Nacional de Investigación (CNI) y la Oficina de Seguridad del Ministerio de Educación trabajaron en la desarticulación y eliminación de toda postura disidente al Régimen de aquellos considerados "enemigos marxistas internos". Además, se estructuró una amplia campaña de capacitación a docentes, directivos y estudiantes para fidelizarlos a las políticas educativas y sociales impuestas (RUIZ, 2010; WEIBEL, 2013).

Esta política de persecución estuvo también presente en la educación superior que se intensificó en el año 1979 debido a la emergencia de un movimiento social contrario a los cambios que habían acontecidos (WEIBEL, 2013). Ejemplo de lo dicho es el reemplazo de rectores por autoridades designadas y la exoneración de multitud de profesores de universidad estatales por ser considerados un riesgo para la estabilidad nacional.

Sin embargo, no es sino hasta 1979, segundo periodo señalado por Ruiz (1997) y Corvalán (2013), en donde el gobierno se decide a restructurar el sistema educacional, e instalar definitivamente las políticas neoliberales ya mencionadas. La privatización del sistema educativo, a través de un Estado cuyo papel "[...] no es otro que el de un ente meramente normativo y supervisor" (RUIZ, 1997, p. 4) y que para Oliva (2008) implica la consolidación y apertura definitiva a 
la privatización de la educación con recursos públicos, idea pensada desde los inicios de la dictadura militar.

El rol del Estado en educación consiste en: fomentar la iniciativa de las organizaciones privadas para que intervengan progresivamente en la gestión educacional, bajo orientación y apoyo de los órganos técnicos del Ministerio de Educación (MINEDUC, 1975-1976, citado por OLIVA, 2008, p. 218).

La autora señala que el motor de estos cambios es sin duda la constitución de 1980, en la cual el Régimen Militar da cabida explícita a la libertad de enseñanza. A partir de aquí pasa de ser un bien público a transformarse en un bien de consumo:

La libertad de enseñanza incluye el derecho de abrir, organizar y mantener establecimientos educacionales. La libertad de enseñanza no tiene otras limitaciones que las impuestas por la moral, las buenas costumbres, el orden público y la seguridad nacional. La enseñanza reconocida oficialmente no podrá orientarse a propagar tendencia político partidista alguna. Los padres tendrán el derecho de escoger el establecimiento de enseñanza para sus hijos (CHILE, 2002, p. 13).

En este sentido, un hito importante es la descentralización educativa a través de la municipalización, con lo cual se inicia, formalmente, el desmantelamiento del sistema educativo anterior (NUÑEZ et al., 1984; RUIZ, 1997, 2010; NÚÑEZ; WEINSTEIN; MUÑOZ, 2010; CASTRO-PAREDES, 2012; CORVALAN, 2013). Es entonces cuando el Estado se desliga de su responsabilidad administrativa y la traspasa a los diferentes municipios y a los sostenedores particulares. El Ministerio de Educación tendrá como única responsabilidad el velar por los temas curriculares y de cumplimiento de asistencia de los estudiantes (OLIVA, 2010).

A esta situación le acompaña otra señal importante. El Decreto con Fuerza de Ley DLF. N. ${ }^{\circ}$ 1-3063 de 1980 presenta en su artículo 4. ${ }^{\circ}$, además de anunciar la lógica de descentralización, que el Código del Trabajo y de Previsión se regirán por lo planteado en el sector privado, quedando a criterio de sostenedores y municipales el funcionamiento de los centros educativos. Con 
ello, se produce una disminución significativa de los salarios profesionales, aumentando la inseguridad laboral y la desvalorización del colectivo docente. Esto tiene, sin duda alguna, una fuerte repercusión tanto en los docentes como en los directivos, quienes dejarían de ser funcionarios públicos y, por tanto, se estaría dando término a la carrera funcionaria al interior del Ministerio de Educación (NÚÑEZ et al., 1984; RUIZ, 1997, 2010). Sin perjuicio de lo anterior, el Gobierno Militar promete y proyecta, desde entonces, la elaboración de un Estatuto Docente que se legisló, finalmente, con aprobación de la Junta Militar, en el año 1987 (NÚÑEZ; WEINSTEIN; MUÑOZ, 2010).

Junto a la municipalización ya comentada, con las consecuencias propias de reproducción de la inequidad - desigualdad y disminución de la calidad del sistema escolar -, el Gobierno Militar propuso la flexibilización de los planes y programas de la Educación General Básica, señalando la importancia de considerar los diversos contextos sociales y los diferentes recursos económicos presentes en las variadas zonas del país para su aplicación. Se autoriza, a través del Decreto con Fuerza de Ley, DFL N. ${ }^{\circ} 4002$ de 1980, la disminución de horas de trabajo y eliminación de alguna asignatura por imposibilidad de cobertura debido a causas económicas. Este Decreto sienta, también, las bases filosóficas y paradigmáticas de las teorías pedagógicas implantadas en el currículo escolar (CAZANGA, 2013). Este cambio fue sustancial pues comenzó a marcar las diferencias entre la educación pública y privada, entre los que tienen más y los que tienen menos, debido a que los planes y programas se transformaron en el mínimo obligatorio para las instituciones con más recursos, las privadas, y el "techo" para las más desfavorecidas, las públicas.

Se tecnocratizó el currículo escolar dando paso a una pedagogía por objetivos, noción educativa articulada y orientada bajo los postulados de la teoría del capital humano (CORVALÁN, 2013), lo que para Oliva (2010, p. 318) se constituyó en la "arquitectura del orden neoliberal", añadiendo que:

La trama de la política curricular de la dictadura es la de la profundización del currículum técnico que, mediante la pedagogía por objetivos, constituye el dispositivo que articula el conjunto de cambios mediante los cuales se implanta la idea de subsidiariedad del Estado.

Respecto a la Educación Superior, se dictaron una serie de Decretos con Fuerza de Ley para reestructurarla drásticamente (DFL N. ${ }^{\circ} 1$ y 4 de 1980; y DFL N. ${ }^{\circ} 5$ y 24 de 1981). Entre dichas medidas, junto al término de la gratuidad de los estudios (RUIZ, 1997, 2010), destaca la eliminación del carácter universitario 
de las carreras pedagógicas, lo que sin duda tuvo consecuencias en relación al menoscabo de la profesión y a la calidad del proceso formativo.

Junto a lo anterior, a partir de las sedes regionales de las Universidades ubicadas en Santiago (Universidad de Chile y Universidad Técnica del Estado) se crearon nuevas universidades públicas, en un principio, y otras de carácter privado posteriormente, lo que sin duda contribuyó a la descentralización educativa que se proponía y que era prioridad para el Régimen Militar. De las Facultades de Educación se formaron dos Academias Superiores de Ciencias Pedagógicas, que son las actuales Universidad Metropolitana de Ciencias de la Educación y la Universidad de Playa Ancha (CAZANGA, 2013).

Finalmente, un elemento que a nuestro parecer es significativo en cuanto a consecuencias del estado actual de la educación universitaria en Chile, es que en esta reestructuración de la educación superior el gobierno da amplias facilidades y garantías para la creación de centros de formación técnica, institutos superiores y universidades de carácter privado, con muy poca o escasa supervisión por parte del Ministerio de Educación, y por ende, del Estado (COX, 1989). Situación que desemboca en 1998 con un sistema gibarizado con 66 universidades, de las cuales sólo 25 reciben aporte estatal, grupo en que coexisten instituciones estatales y privadas, pero privadas que pertenecen a un selecto grupo de universidades denominadas tradicionales, que se les reconoce su calidad y vocación de servicio público.

Se trata, en definitiva, de transferir la educación de la esfera de la política a la del mercado, negando su condición (real o hipotética) de derecho social y transformándola en una posibilidad de consumo individual, variable según el mérito y capacidad de los consumidores (GENTILI, 1997, p. 60).

\section{La década de 1990: sistema educativo-escolar tras la vuelta de la democracia}

En Chile, en el proceso de transición hacia la democracia no fue fácil transitar por las modificaciones en las políticas educativas. En ese complejo devenir, destacamos tres elementos centrales que describimos a continuación.

a) Enclave autoritario, herencia de la dictadura. El gobierno militar cerró todo proceso de reestructuración del sistema educativo a través de la Ley N. ${ }^{\circ}$ 18.962 (LOCE: Ley Orgánica Constitucional de la Enseñanza), publicada el 10 
de marzo de 1990, justo antes de terminar su mandato (OLIVA, 2008). Dicha ley permitió proteger todas las políticas impuestas, que queda resguardada por la Constitución Política de 1980, el mayor enclave autoritario de la época (RUIZ, 1997). La LOCE fue "[...] el camino usado por la dictadura para perpetuar su arquitectura educativa neoliberal" (OLIVA, 2010, p. 317).

b) Decisiones político-estratégicas tomadas por la Concertación de Partidos por la Democracia. Esta colectividad asume el gobierno tras la vuelta a la democracia. En esos momentos se decidió no revertir la municipalización que vivía el sistema educativo y continuar con el sistema de financiamiento vigente. Ello tras un implícito acuerdo entre los dos grandes conglomerados políticos, el de centro-derecha (Alianza por Chile) y el de centro-izquierda (Concertación de Partidos por la Democracia). Ello permitiría, según actores de dicho acuerdo, no romper el equilibrio político ya reestablecido (OCDE, 2004, en OLIVA, 2008).

c) Rehuir toda discusión política relacionada con una reforma educacional postdictadura. Se asumió la LOCE en su totalidad, postergándose la reflexión acerca de posibles cambios en la estructura educativa vigente. Los discursos tecnocráticos y economicistas, junto a los conceptos de competencia, eficiencia y eficacia comenzaron a ser protagonistas relevantes en el sistema educativo (ALARCÓN, 2012). Esta situación queda de manifiesto en la siguiente declaración del Ministro de Educación de la época: "La formulación de políticas de largo plazo que no afecten brusca y traumáticamente a los actores del sistema educativo, que recoja la tradición y la innovación, debe hacerse a partir de la realidad que heredamos" (LAGOS, 1990, en NÚÑEZ, 1995, p. 178).

La Concertación de Partidos por la Democracia no sólo no detuvo la tendencia economicista de las políticas instauradas durante la dictadura, sino que las acentuó, proponiendo modificaciones curriculares sin penetrar en la estructura profunda que mantenía, y mantiene, un sistema educativo desigual y segregador (COZANGA, 2013; RUIZ, 1997). Se mantiene la municipalización, el modelo de financiamiento, y los profesores no recuperan su condición de empleados públicos.

A partir de esta conclusión podemos reafirmar nuestro aserto en cuanto a afirmar que la reforma pedagógica de la Concertación debe ser considerada más bien como un cambio cosmético de un modelo educacional diseñado para servir de apoyo ideológico al modelo de desarrollo económico neoliberal o de libre mercado, por los sectores que sirvieron de sustentación social a la Dictadura (CAZANGA, 2013, p. 1). 
Bajo esta situación, es importante relevar como 'enclave autoritario' de la dictadura, que la LOCE es una Ley Constitucional que requiere de un alto quorum en ambas cámaras del Congreso Nacional para su modificación. Es así como en el año 2009, 20 años después, se consigue derogar la LOCE y se promulga la Ley General de Educación (LGE) la cual no fue bien recepcionada por varios sectores relacionados con el ámbito educativo, y que para Oliva (2010, p. 317) es un cuerpo legal en el que "[...] permanece incólume el rol del Estado subsidiario, piedra angular de la gran ofensiva neoliberal".

A pesar de lo dicho, no es fácil negar los intentos de transformación llevados a cabo por la Concertación de Partidos por la Democracia. Entre ellos podemos destacar los siguientes (COX, 2005):

- Construcción de un discurso social y pedagógico que releva el rol de la educación en la construcción de una sociedad más justa.

- Énfasis en los conceptos de calidad y equidad como ejes vertebradores del sistema educativo.

- Implementación de programas integrales para lograr mayor cobertura educativa y otorgar mayores recursos a los centros educativos más desfavorecidos.

- Proyección de un sostenido aumento en el presupuesto nacional para el sistema educativo.

- Los profesores pasan a ser regidos por el Estatuto Docente y no por el código del trabajo del sector privado.

- Reestructuración del curriculum escolar, intentando transitar desde un enfoque centrado en la "pedagogía por objetivos" hacia un modelo comprensivo de la enseñanza y el aprendizaje.

Estos intentos estuvieron acompañados por una aspiración de corregir la desigualdad ocasionada por la lógica de la dictadura militar basada en el sistema de mercado (CARNOY, 2010a; OLIVA, 2008). Para ello facilitaron recursos, a manera de compensación, a las escuelas rurales, a las escuelas primarias más desfavorecidas, a las secundarias con bajos ingresos y un sistema de préstamos a los estudiantes universitarios, además de la generación de un programa nacional más coherente y homogéneo, la instalación de la jornada escolar completa, otorgamiento de libros de textos con carácter gratuito, una red de conexión a Internet (Red Enlaces), siempre con una intencionalidad de igualdad para todos y todas.

En los años 1990, con la intención de obtener información que pudiera ayudar en la mejora y equidad del sistema educativo chileno, se optó por un sistema de evaluación basado en los exámenes nacionales (SIMCE en 1988: Sistema de Evaluación de la Calidad Educativa). Pero lo que nació con una intencionalidad evaluativa de mejora pareciera que no ha cumplido con su objetivo y pareciera haberse convertido en un sistema a partir del cual detectar 
las escuelas con bajo rendimiento académico y estigmatizarlas. Una muestra clara de ello es que el gobierno decidió emplear un sistema a partir del cual se construiría un mapa de las escuelas chilenas identificándolas con semáforos de color rojo, ámbar y verde en clara alusión a escuelas con bajos rendimientos, medianos rendimientos y buenos rendimientos ${ }^{4}$. Este mapa sería entregado a las escuelas y a los padres y madres para que conocieran, de primera mano, las escuelas a las que asisten sus hijos (MINEDUC, 2010).

El criterio de competitividad propio de las políticas neoconservadoras del gobierno militar aún hace presencia en el sistema educativo actual. El SIMCE junto con los exámenes de carácter internacional (Estudio Internacional de Tendencias en Matemática y Ciencias - TIMSS, Programa Internacional de Evaluación de los Alumnos - PISA) dan clara muestra de la dirección tomada por el país en lo que a educación se refiere. Las críticas, tras los resultados mostrados por las diferentes pruebas, no tardaron en llegar y posicionarse en el inconsciente colectivo de la ciudadanía, siendo sinónimo de movimientos críticos de resistencia que han alzado la voz pero que no parecen haber conseguido profundos cambios en la lógica que mantiene dicho sistema.

El economista de la educación, Martin Carnoy, mantiene que a pesar de los esfuerzos por los gobiernos democráticos actuales, el " [...] sistema de mercado educativo heredado de los militares siguió teniendo influencia en las políticas educativas chilenas en la década de los noventa, mucho más que en cualquier otro país latinoamericano" (CARNOY, 2010a, p. 47). Una muestra clara de ello es que el $47 \%$ de los niños, en los años 1990, asistían a escuelas privadas o particulares subvencionadas, y esta proporción sigue aumentando. Y el 70\% del costo de a educación superior sigue siendo financiado por las familias de los estudiantes. Incluso, se les ha permitido a las escuelas, independientemente de su dependencia administrativa, seleccionar a sus estudiantes y cobrarles una colegiatura máxima de 150 dólares como máximo, y ello no significa perder la subvención (CARNOY, 2010a).

\section{El siglo XXI y la construcción de un sistema educativo desigual}

Si bien los intentos transformativos han sido reales, y algunos de ellos se han podido operacionalizar, la realidad actual no dista mucho de lo contado hasta aquí. El sistema educativo-escolar se sigue dividiendo en escuelas priva-

4 Afortunadamente esta propuesta no prosperó. 
das, particulares subvencionadas y en municipales. Y los padres tenían y tienen la potestad de elegir a qué escuelas asisten sus hijos. Pero es curioso que las escuelas privadas y las municipales reciben similares recursos desde el gobierno central, atendiendo siempre a un criterio de promedio de asistencia de estudiantes a dichas escuelas, desembocando, claro está, en un sistema educativo en el que escuelas privadas y municipales compiten por el mismo alumnado. Esta lógica también sustenta el mundo universitario, contexto en el que el estudiantado y sus familias financian casi completamente el proceso de formación.

Para nadie es desconocida la situación de desigualdad extrema que vive Chile en estos momentos (LÓPEZ; FIGUEROA; GUTIÉRREZ, 2013; MAYOL, 2012; CARNOY, 2010b). La OCDE (2013) señala que Chile es el país más desigual de todos los que conforman dicha organización, explicitando que el $10 \%$ más rico de la población posee un ingreso económico 27 veces mayor que el 10\% más pobre. Pero la desigualdad no se centra, sólo, en los ingresos económicos, sino también en términos sociales y educativos, ámbito éste último que nos ocupa. Una muestra clara de ello es que en el año 2008 sólo el $43 \%$ de los estudiantes asistían al sistema público de enseñanza y que la educación privada con subvención estatal ocupa, en la actualidad, casi la mitad de la matrícula total en el sistema educativo formal (DONOSO; ARIAS, 2012). Chile posee un sistema educativo desigual y segregacionista (VILLALOBOS; VALENZUELA, 2012), traducido en escuelas para pobres (municipales), escuela para la clase media (particulares subvencionadas) y escuela para ricos (particulares pagadas) (PUGA, 2011).

Esta desigual situación se ha traducido, también, en término de resultados académicos obtenidos por los estudiantes (Tablas 1 y 2 ).

TABLA 1 - RESULTADOS 4. ${ }^{\circ}$ AÑO BÁSICO EN LECTURA, MATEMÁTICAS Y CIENCIAS NATURALES SEGÚN GRUPO SOCIOECONÓMICO

\begin{tabular}{|c|c|c|c|}
\hline \multirow{2}{*}{ Grupo Socioeconómocio } & \multicolumn{3}{|c|}{ Promedios 2011 } \\
\cline { 2 - 4 } & Lectura & Matemáticas & Ciencias Naturales \\
\hline Bajo & 249 & 235 & 234 \\
\hline Medio Bajo & 253 & 243 & 243 \\
\hline Medio & 267 & 259 & 260 \\
\hline Medio Alto & 284 & 278 & 280 \\
\hline Alto & 299 & 301 & 297 \\
\hline
\end{tabular}

FUENTE: Modificado de SIMCE (2011). 
TABLA 2 - RESULTADOS 8. ${ }^{\circ}$ AÑO BÁSICO EN LECTURA, MATEMÁTICAS, CIENCIAS NATURALES Y CIENCIAS SOCIALES SEGÚN DEPENDENCIAADMINISTRATIVA DEL ESTABLECIMIENTO EDUCACIONAL AL QUE ASISTEN

\begin{tabular}{|c|c|c|c|c|}
\hline Dependencia & \multicolumn{4}{|c|}{ Promedios 2011 } \\
$\begin{array}{c}\text { Administrativa } \\
\text { Establecimientos } \\
\text { Educacionales }\end{array}$ & Lectura & Matemáticas & Ciencias Naturales & Ciencias Sociales \\
\cline { 3 - 5 } Municipal & 240 & 242 & 245 & 244 \\
\hline $\begin{array}{c}\text { Particular } \\
\text { Subvencionado }\end{array}$ & 259 & 265 & 270 & 267 \\
\hline Particular Pagado & 293 & 311 & 309 & 306 \\
\hline
\end{tabular}

FUENTE: Modificado de SIMCE (2011).

Todo esto ha desembocado en perversas consecuencias. El sector municipal da muestras de una fuerte fase de decrecimiento en los últimos años, mientras que el sector particular recibe a los alumnos que abandonan la enseñanza municipal. Si en 1990 los colegios municipales representaban un 61,2\% de la matrícula de enseñanza básica, aproximadamente 30 puntos porcentuales por arriba del sector particular subvencionado (31,8\%), en el año 2007 esa distancia se habría reducido a 4,3 puntos porcentuales. Algo similar ocurre en la enseñanza media, en donde el sector municipal representaba un 55,3\% de las matrículas en 1990, en comparación con el $35,4 \%$ de las escuelas particulares subvencionadas y un 9,3 de las particulares pagadas. En el año 2007 la matrícula se repartía en tres partes relativamente iguales, lo que sin duda refleja un migración de estudiantes desde los establecimientos municipales a los particulares subvencionados (MARCEL; RACZYNSKI, 2009).

Recientemente, en el año 2008, se aprobó la ley de subvención preferencial (N. ${ }^{\circ}$ 4030-04), es decir, que los establecimientos educacionales no recibirán dicha subvención en función del número de niños y de la asistencia a clases, sino que ésta será entregada atendiendo también a la situación de vulnerabilidad de los estudiantes, es decir, que las instituciones con mayor número de niños en situación de vulnerabilidad económica y social recibirán montos mayores, lo que se pretende pueda paliar un poco las desigualdades económicas y materiales que existen por escuela (OPECH, 2008).

Pero claro está que a pesar de los cambios realizados a la ley, la lógica del sistema de financiamiento de la educación chilena se mantiene en lo sustancial pues las subvenciones seguirán siendo entregadas en función de la demanda y los alumnos, aunque ahora será por alumnos en situación de vulnerabilidad, lo que sin duda no resuelve el problema de inestabilidad económica de las escuelas chilenas. 
El Estado mantiene su rol subsidiario, entregando a los sostenedores la voluntariedad de incorporarse al sistema, suscribiendo con el Ministerio de Educación un "Convenio de Igualdad de Oportunidades y Excelencia Educativa", lo que hará que solo algunas escuelas se acojan a este convenio, solo regulado por los resultados SIMCE. Esto significa que el Estado en la práctica no asume la responsabilidad en la gestión pedagógica de la totalidad del sistema educativo público, hoy en crisis, dejando esto a cada sostenedor y al SIMCE (OPECH, 2008, p. 1).

La supervisión se focaliza, entonces, sobre aquellos establecimientos educativos denominados como prioritarios, dejando sin intervención estatal a aquellos centros que no se atengan a la firma de los mencionados convenios. También es importante mencionar que se mantendrá el mismo sistema de evaluación de la calidad, SIMCE (OEPCH, 2008), aún sabiendo que el mismo sólo atiende a algunos sectores de aprendizaje (matemáticas y lenguaje principalmente) por lo que las mejoras derivadas de dicha evaluación sólo irá direccionada a estos sectores, obviando otros que contribuyen tanto o más a la calidad educativa integral de los educandos.

A pesar de lo dicho, es realmente sorprendente constatar que Chile es uno de los países con mayor gasto total en educación, superando en 0,9 puntos porcentuales el promedio de los países de la Organización para la Cooperación y el Desarrollo Económico (OECD) y sobrepasando largamente a países como Irlanda, España y Japón. El problema chileno no está tanto en el gasto total en educación como \% del Producto Interno Bruto (PIB), sino que la verdadera lacra está cuando se descompone la cifra de ese gasto total, puesto que un $48 \%$ de ese gasto $\left(3,25 \%\right.$ del PIB) proviene de fuentes privadas ${ }^{5}$, siendo Chile el país que tiene un mayor nivel de gasto privado como porcentaje del PIB aporta al gasto total y uno de los países con menor gasto público en educación. El gasto privado en educación como \% del PIB es casi cinco veces más alto que el promedio de los países de la OECD, siendo exactamente de 1,7 puntos porcentuales más bajo que el promedio de los mismos países (KREMERMAN, 2007, p. 76).

Las diferencias siguen siendo significativas. Casi un tercio de lo que se gasta en educación básica y media proviene de las propias familias chilenas, mientras que en los países de la OECD sólo un 7,3\% proviene de las familias.

A otros enseñaron secretos que a ti no / a otros dieron de verdad esa cosa llamada educación / ellos pedían esfuerzo, ellos pedían dedicación

5 Cuando hablamos de gasto privado estamos haciendo referencia, principalmente, al gasto que realizan las familias de esos niños y niñas que pertenecen al sistema educativo chileno. 
/ ¿y para qué? para terminar bailando y pateando piedras (LOS PRISIONEROS, 1986).

\section{Conclusiones}

Es nuestro deseo terminar este trabajo con una reflexión que invite a un trabajo pedagógico transformativo dirigido hacia mayores cuotas de justicia social. Es por ello que aprovechamos las palabras de Eduardo Galeano, al decirnos:

Un viejo proverbio enseña que mejor que dar pescado es enseñar a pescar. El obispo Pedro Casaldáliga, que no nació en América pero la conoce por dentro, dice que sí, que eso está muy bien, muy buena idea, pero ¿qué pasa si nos envenenan el río? ¿O si alguien compra el río, que era de todos, y nos prohíbe pescar? O sea: ¿qué pasa si pasa lo que está pasando? La educación no alcanza.

\section{REFERENCIAS}

ALARCON, C. Aperturas. Propuesta Educativa, Buenos Aires, v. 38, n. 2, p. 32-38, Nov. 2012.

CARNOY, M. La competencia en Educación es una locura de la derecha. Santiago de Chile, 2010a. Entrevista realizada por Jaime Retamal en el diario electrónico elmostrador. cl. Disponible en: $<$ http://www.elmostrador.cl/noticias/pais/2010/06/24/\%E2\%80\%9Cla-competencia-en-educacion-es-una-locura-de-la-derecha $\% \mathrm{E} 2 \% 80 \% 9 \mathrm{D} />$. Consultado en: 03/08/2010.

. La ventaja académica de Cuba. ¿Por qué los estudiantes cubanos rinden más? México: Fondo de Cultura Económica, 2010b.

CASTRO-PAREDES, M. Descentralización educacional en Chile: itinerario sin territorio. Innovar, Bogotá, v. 22, n. 43, p. 77-92, Ene-Mar. 2012.

CAZANGA, O. Crisis de la educación en Chile: 1973-2000. Santiago, 2013. Disponible en: $<$ http://www.fortinmapocho.com/detalle.asp?iPro=791\&iType=126>. Consultado en: $15 / 11 / 2013$. 
CHILE. Constitución Política de la República de Chile 1980 (8va. ed). Santiago: Jurídica de Chile, 2002.

CORVALAN, C. La narrativa educacional chilena y su proceso de transformación reciente: un análisis sociológico-histórico. Folios, Bogotá, n. 37, p. 63-81, 1er Sem. 2013.

COX, C. Autoritarismo, mercados y conocimiento: evaluación de las políticas de educación superior en Chile en los 80. Santiago: CIDE, 1989.

. Las políticas educacionales de Chile en las últimas dos décadas del siglo XX. En: . Politicas educacionales en el cambio de siglo: La reforma del sistema escolar de Chile. Santiago: Universitaria, 2005. p. 19-113.

DONOSO, S.; ARIAS, O. Distribución desigual de las oportunidades educativas en el territorio y migración de la matrícula escolar: el caso de la región de Los Lagos (Chile). Estudios Pedagógicos, Valdivia, v. 38, n. 2, p. 5-54, Ago-Dic. 2012.

GENTILI, P. El consenso de Washington y la crisis de la educación en América Latina. Archipiélago: Cuadernos de Crítica de la Cultura, Barcelona, n. 29, p. 56-65, Abr-Jun. 1997.

KREMERMAN, M. Radiografía del Financiamiento de la Educación Chilena: Diagnóstico, Análisis y propuestas. Santiago de Chile, 2007. Disponible en: <http://www. opech.cl/inv/investigaciones/Kremerman_Radiografia_Financiamiento_Educacion. pdf>. Consultado en: 30/06/2010.

LÓPEZ, R.; FIGUEROA, E.; GUTIÉRREZ, P. La 'parte del león': Nuevas estimaciones de la participación de los súper ricos en el ingreso de Chile. Serie de Documentos de Trabajo. Santiago de Chile: Departamento Economía, Universidad de Chile, 2013. Disponible en: <http://www.econ.uchile.cl/descargar/publicacion/la-parte-del-leon-nuevas-estimaciones-de-la-participacion-de-los-super-ricos-en-el-ingreso-de-chile $>$. Consultado en: 09/07/2013.

LOS PRISIONEROS. “El baile de los que sobran”. En: Pateando Piedras. Emi Odeon Chileba, 1986. CD.

MARCEL, M.; RACZYNSKI, D. La asignatura pendiente. Claves para la revalidación de la educación pública de gestión local en Chile. Santiago de Chile: Uqbar Editores, 2009.

MAYOL, A. El derrumbe del modelo. La crisis de la economía de mercado en el Chile contemporáneo. Santiago de Chile: LOM Ediciones, 2012.

MINEDUC. Planes y programas de estudio. Santiago de Chile, 2010. Disponible en: $<$ www.mineduc.cl $>$. Consultado en: 14/01/2010.

NUÑEZ, I. Hacia un nuevo paradigma de reformas educativas: la experiencia chilena. Pensamiento Educativo, Santiago, v. 17, n. 2, p. 173-189, Ago-Dic. 1995.

NUÑEZ, I. et. al. Las transformaciones de la educación bajo el régimen militar. Santiago, PIIE, 1984. v. 1. 
NUÑEZ, I.; WEINSTEIN, J.; MUÑOZ, G. ¿Posición olvidada?: Una mirada desde la normativa a la historia de la dirección escolar en Chile (1929-2009). Psicoperspectivas. Individuo y Sociedad, Valparaíso, v. 9, n. 2, p. 53-81, Jul-Dic. 2010.

OCDE. Economic Policy Reforms 2013: Going for Growth. EEUU: OEDC Publishing. Disponible en: < http://dx.doi.org/10.1787/growth-2013-3n>. Consultado en: 09/07/2013.

OLIVA, M. Política educativa chilena 1965-2009. ¿Qué oculta esa trama? Revista Brasileira de Educação, Rio de Janeiro, v. 15, n. 44, p. 311-328, May-Ago. 2010.

. Política educativa y profundización de la desigualdad en Chile. Estudios Pedagógicos, Valdivia, v. 34, n. 2, p. 207-226, Ago/Dic. 2008.

OPECH. Documento de trabajo N. ${ }^{\circ} 2$. ¿Ley subvención escolar preferencia? Notas para la discusión. 2008. Disponible en: <http://www.opech.cl/inv/ documentos_trabajo/ LEY\%20\%20SUBVENCI\%C3\%93N\%20ESCOLAR\%20PREFERENCIAL_Corregido_Versi $\%$ C3\%B3n\%20\%20Final.pdf>. Consultado en: 13/03/2009.

PUGA, I. Escuela y estratificación social en Chile: ¿cuál es el rol de la municipalización y la educación particular subvencionada en la reproducción de la desigualdad social? Estudios Pedagógicos, Valdivia, v. 37, n. 2, p. 213-232, Ago.-Dic. 2011.

RIESCO, M. Se derrumba un mito: Chile reforma sus sistemas privatizados de educación y previsión. Santiago: CENDA, 2007.

RUIZ, C. De la república al mercado: Ideas educacionales y política en Chile. Santiago: LUM, 2010.

. Educación, Mercado y Privatización. Santiago, 1997. Disponible en: <web. uchile.cl/facultades/filosofia/Editorial/documenta/reflexunive/08.htm $>$. Consultado en: $15 / 11 / 2013$.

SIMCE. Resultados SIMCE 2011. 4. ${ }^{\circ}$ y $8 .^{\circ}$ básico. Santiago de Chile: Ministerio de Educación, 2011.

VILLALOBOS, C.; VALENZUELA, J. Polarización y cohesión social del sistema escolar chileno. Revista de Análisis Económico, Geogetown, v. 27, n. 2, p. 145-172, Ago.-Dic. 2012.

WEIBEL, M. Así nació la educación de Pinochet. Santiago de Chile, 2013. Disponible en: < http://www.theclinic.cl/2013/06/27/asi-nacio-la-educacion-de-pinochet/>. Consultado en: $15 / 11 / 2013$.

Texto recebido em 18 de janeiro de 2014. Texto aprovado em 07 de fevereiro de 2014. 